\title{
Optic Nerve Disorder
}

National Cancer Institute

\section{Source}

National Cancer Institute. Optic Nerve Disorder. NCI Thesaurus. Code C79698.

A non-neoplastic or neoplastic disorder affecting the optic nerve (second cranial nerve). 